\title{
Synaptopathies, Neurotransmitters and Pharmacological Management of Epilepsy and Neurodevelopmental Disorders
}

\author{
Javier López Pisón* and Marta Marín Andres \\ Neurometabolic Section, Hospital Miguel Servet, Zaragoza, Spain
}

*Corresponding author: Javier López Pisón, Chief of Section Neurometabolic Section, Pediatric Department, Hospital Miguel Servet, Zaragoza, Spain

\section{Opinion}

We pediatric neurologists treat many children with neurodevelopmental disorders and/or epilepsy. The pharmacological treatment of epilepsy, so often ineffective and with many side effects, is not commonly discussed. However, the pharmacological treatment of neurodevelopmental disorders, particularly of attention deficit disorders either with or without hyperactivity (ADD/ADHD) is very much discussed, despite so much evidence regarding its effectiveness. Pharmacological treatment of epilepsy is not a cure, nor in many cases does the long-term evolution of epilepsy or neurodevelopment change. Childhood epilepsy is refractory to antiepileptic drugs (AEDs) in around twenty per cent of cases. Some AEDs can cause or worsen ADD/ADHD symptoms and in some cases should be discontinued [1]. The certainty of epilepsy diagnosis and the frequency, intensity, duration and impact of seizures should be valued to establish treatment. It is important to make sure that treatment is well tolerated and to be alert to any possible side effects, especially those affecting attention, behavior or school performance.

ADD/ADHD figures among children with epilepsy are between $16.7 \%$ and $50 \%$, influenced by AEDs, the type of epilepsy and the underlying brain impairment [2,3]. In certain cases, attention deficit may improve with treatment for epilepsy but, if this is not the case, treatment for ADD/ADHD should be considered. There are numerous references to the effectiveness and safety of treating children with epilepsy and ADD/ADHD with stimulants $[3,4]$. When making decisions regarding the treatment of children with epilepsy, it should be considered that in the long term, the child and his neurodevelopment are more important than epilepsy. Neurodevelopmental disorders, that include intellectual disability
(ID), autism spectrum disorder (ASD), ADD/ADHD, tic disorders and learning disability, have a high prevalence and a significant personal, family and social impact. They can occur isolated or associated, and are associated with various brain problems, both inherited or acquired [5].

ID, ASD and ADD/ADHD and many cases of other neurodevelopmental disorders share executive brain dysfunction. Executive brain dysfunction is associated with a lot of neurological pathology in children: neurodevelopmental disorders, epilepsy, acquired brain injury [6], neurofibromatosis type 1 [7], tuberous sclerosis [8], Duchenne muscular dystrophy [9], cerebral palsy, or neurometabolic diseases. Cognitive and attention functions are not located solely in the frontal lobe and they depend on adequate neuronal circuits throughout the brain [10], which explains the high prevalence of ADD/ADHD in different encephalopathies .

No diagnosis can exclude ADD/ADHD. Executive brain dysfunction occurs in people of normal intelligence, with ID and with high capacities. It can be associated with ASD and Asperger syndrome. It can be accompanied by tics, oppositional-defiant disorder, depression, anxiety and with learning disorders. It can cause headaches.

ADHD goes undiagnosed in a considerable number of children and adolescents [11], particularly in girls and women [6].

Neurodevelopmental disorders are not categorical diagnoses and are evolving; it is well known that over the years they tend to improve the autistic characteristics [12] and nuclear symptoms of ADD/ADHD. Therefore, prognosis is uncertain in neurodevelopmental disorders, more so with the youngest children. Prognosis is also influenced by therapeutic interventions, 
including drug treatment. Executive brain dysfunction has effective drug treatment. Psychostimulants are effective in $80 \%$ of cases of ADD/ADHD. They help regulate the correct functioning of brain neurotransmitters, necessary for the executive brain function. Response can be very positive and can be observed from the beginning of treatment: changes the child's life, improves school performance, social relationships and family coexistence, time spent on sport and hobbies, and self-esteem. If stimulants fail to work or are not well tolerated, other drugs exist that can be effective and need to be tested.

The NICE Guideline of 2018 indicates that ADHD should be considered in all age groups with symptom criteria adjusted for age-appropriate changes in behaviour and that medication must be offered to children aged 5 years and over if their ADHD symptoms are still causing a persistent significant impairment in at least one domain after environmental modifications have been implemented [6].

The Clinical Practice Guideline from the American Academy of Pediatrics of 2019 indicates that the pediatrician should initiate an evaluation for ADHD for any child or adolescent over 4 years old who presents academic or behavioral problems and symptoms of inattention, hyperactivity, or impulsivity, that treatments available have good evidence of efficacy and a lack of treatment means risking impaired outcomes, and that diagnostic criteria for ADHD can be applied to preschool-aged children (4-6 Years). The American Guide also advises caution with treatment for children 4 to 5 years of age as evidence suggests that the rate of metabolizing methylphenidate is slower and a child's response to stimulants is variable and unpredictable [11]. However, contrary to all evidence, over $20 \%$ of our patients diagnosed with ADD/ADHD received no pharmacological treatment, and 30\% delayed the start of treatment an average of 20 months [5]. Once treatment is initiated, adherence is high $[5,13]$.

A person's decision to start, change or stop treatment may be influenced by media coverage, teachers, family members, friends and differing opinion on the validity of a diagnosis of ADHD [6]. Cortes-Saladefont, et al. indicate that neurodevelopmental disorders, such as epilepsy and movement disorders, are frequently a sign of synaptopathies and there are treatment prospects aimed at regulating the function of neurotransmitters in the synaptic vesicles [14]. We already have drugs that improve brain executive function. It is our responsibility to offer them.

\section{References}

1. Garzon P, Lemelle L, Auvin S (2016) Childhood absence epilepsy. An update. Arch Pediatr 23(11): 1176-1183.

2. Costa CR, Oliveira GM, Gomes M, Maia Filho HS (2015) Clinical and neuropsychological assessment of attention and ADHD comorbidity in a sample of children and adolescents with idiopathic epilepsy. Arq Neuropsiquiatr 73(2): 96-103.

3. Besag F, Gobbi G, Caplan R, Sillanpää M, Aldenkamp A, et al. (2016) Psychiatric and Behavioural Disorders in Children with Epilepsy (ILAE Task Force Report) Epilepsy and ADHD. Epileptic Disord, Online ahead of print.

4. Ravi M, Ickowicz A (2016) Epilepsy, Attention-Deficit Hyperactivity Disorder and Methylphenidate Critical Examination of Guiding Evidence. J Can Acad Child Adolesc Psychiatry 25(1): 50-58.

5. Marín Andrés M, Gutiérrez Sánchez AM, Martínez de Morentin Navarcorena AL, Bernadó Fonz RA, et al. (2020) Attention deficit disorder with or without hyperactivity isolated in Neuropediatrics consultation. Number of cases. Arch Argent Pediatr 118(4): e405-e409.

6. (2018) Attention deficit hyperactivity disorder: diagnosis and management. NICE guideline [NG87], London.

7. Sanchez-Marco SB, Lopez-Pison J, Serrano-Vinuales I, Troyas-Fernandez de Garayalde L, Lafuente-Hidalgo M, et al. (2019) Neurofibromatosis type 1 and attention-deficit disorder. Our current experience. Nuestra experiencia. Rev Neurol 68(1): 7-10.

8. Wilbur C, Sanguansermsri C, Chable H, Anghelina M, Peinhof S, et al. (2017) Manifestations of Tuberous Sclerosis Complex: The Experience of a Provincial Clinic. Can J Neurol Sci 44(1): 35-43

9. Ricotti V, Mandy WP, Scoto M, Pane M, Deconinck N, et al. (2016) Neurodevelopmental, emotional, and behavioural problems in Duchenne muscular dystrophy in relation to underlying dystrophin gene mutations. Dev Med Child Neurol 58(1): 77-84.

10. Martijn P van den Heuvel, Olaf Sporns (2013) An anatomical substrate for integration among functional networks in human cortex. J Neurosci 33(36):14489-14500.

11. Mark L Wolraich, Joseph F Hagan, Carla Allan, Eugenia Chan, Dale Davison, et al. (2019) Clinical Practice Guideline for the Diagnosis, Evaluation, and Treatment of Attention-Deficit/Hyperactivity Disorder in Children and Adolescents. American Academy of Pediatrics. Pediatrics 144(4): e20192528.

12. Heather A Close, Li-Ching Lee, Christopher N Kaufmann, Andrew W Zimmerman (2012) Co-occuring Conditions and Change in Diagnosis in Autism Spectrum Disorders. Pediatrics 129(2): e305-316.

13. Visser SN, Danielson ML, Bitsko RH, Holbrook JR, Kogan MD, et al. (2014) Trends in the parent-report of health care provider-diagnosed and medicated attention-deficit/hyperactivity disorder: United States, 2003-2011. J Am Acad Child Adolesc Psychiatry 53(1): 34-46.e2.

14. E Cortès Saladelafont, A Tristán Noguero, R Artuch, X Altafaj, A Bayès, et al. (2016) Diseases of the Synaptic Vesicle: A Potential New Group of Neurometabolic Disorders Affecting Neurotransmission. Semin Pediatr Neurol 23(4): 306-320. 
(C) Commons Attribution 4.0 License

To Submit Your Article Click Here: Submit Article

DOI: $10.32474 / 0 J N B D .2020 .04 .000191$

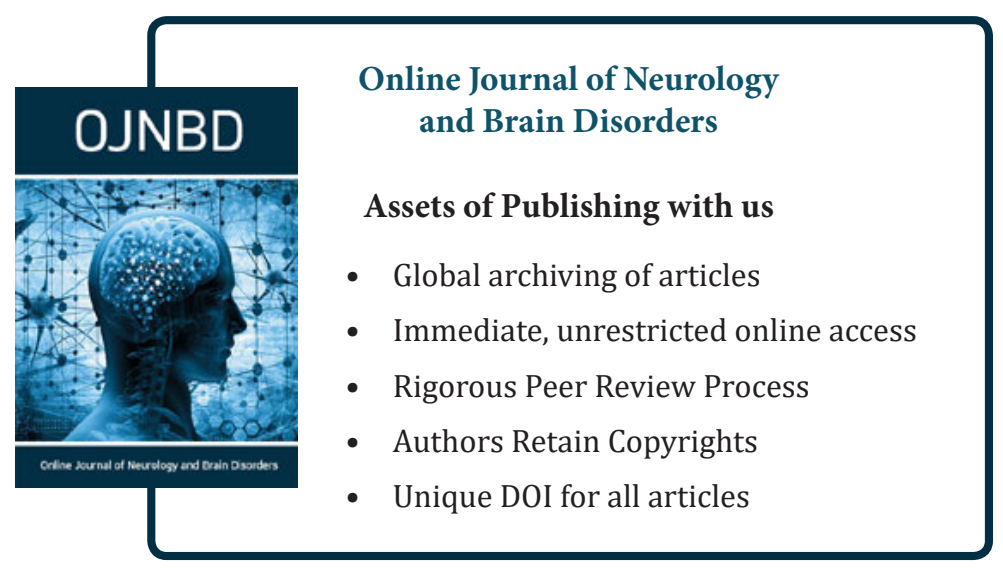

